\title{
1 In search of a core cellular network in single cell transcriptome data
}

2 Ming Yang $^{1}$, Benjamin R. Harrison ${ }^{1}$, Daniel E.L. Promislow ${ }^{1,2^{*}}$

$3{ }^{1}$ Department of Laboratory Medicine and Pathology, University of Washington School of

4 Medicine, Seattle, WA 98195 USA

$5 \quad{ }^{2}$ Department of Biology, University of Washington, Seattle, WA 98195 USA

6

7 *Correspondence:

8 Daniel Promislow

9 promislo@uw.edu 


\section{Abstract}

11 Background: Along with specialized functions, cells of multicellular organisms also perform

12 essential functions common to most if not all cells. Whether diverse cells do this by using the same

13 set of genes, interacting in a fixed coordinated fashion to execute essential functions, remains a

14 central question in biology. Single-cell RNA-sequencing (scRNA-seq) measures gene expression

15 of individual cells, enabling researchers to discover gene expression patterns that contribute to the

16 diversity of cell functions. Current analyses focus primarily on identifying differentially expressed

17 genes across cells. However, patterns of co-expression between genes are probably more indicative

18 of biological processes than are the expression of individual genes. Using single cell transcriptome

19 data from the fly brain, here we focus on gene co-expression to search for a core cellular network.

20 Results: In this study, we constructed cell type-specific gene co-expression networks using single

21 cell transcriptome data of brains from the fruit fly, Drosophila melanogaster. We detected a set of

22 highly coordinated genes preserved across cell types in fly brains and defined this set as the core

23 cellular network. This core is very small compared with cell type-specific gene co-expression

24 networks and shows dense connectivity. Modules within this core are enriched for basic cellular

25 functions, such as translation and ATP metabolic processes, and gene members of these modules

26 have distinct evolutionary signatures.

27 Conclusions: Overall, we demonstrated that a core cellular network exists in diverse cell types of

28 fly brains and this core exhibits unique topological, structural, functional and evolutionary

29 properties. 


\section{Keywords}

31 gene co-expression, co-expression network, core cellular network, single-cell transcriptome,

32 phylostratigraphy, systems biology, Drosophila melanogaster.

\section{Background}

Life on Earth has gone through many transitions in organizational complexity (Smith and

36 Szathmary 1997). Among these, the evolution of multicellularity stands out as a key milestone.

37 This transition has occurred independently multiple times across the tree of life and paved the way

38 for tremendous phenotypic expansion and biological diversification (Parfrey and Lahr 2013).

39 Although this has led to the evolution of cell-type-specific regulatory pathways that define cells

40 with vastly different functions, all cells in multicellular organisms also carry out common

41 functions that are essential for cell survival. Whether these common functions are supported by a

42 common core of genes functioning in all cells, coordinated to ensure survival in the face of diverse

43 functional demands, remains a central question in biology (Lim, Lee, and Tang 2013; Hart and

44 Alon 2013). In particular, do all cells utilize the same set of genes to accomplish common functions,

45 and do these genes function in a fixed and coordinated fashion — a core regulatory network?

46 Cellular phenomena can be characterized by different levels of biological organization, or -

47 omes, such as the genome, epigenome, transcriptome, proteome, etc. Investigating core functions

48 from these different levels not only gives insight into essential functions of cellular life, but also

49 helps to reveal the evolutionary forces acting at different levels of biological organization (Sorrells

50 and Johnson 2015; Ghadie, Coulombe-Huntington, and Xia 2018; Wagner 2012). To identify core 
51 functions at each level, researchers have used various strategies such as identifying constitutively

52 active genes over temporal or spatial scales, and across environments. These genes are typically

53 referred to as 'housekeeping genes' and are thought to perform essential functions. They tend to

54 share aspects of sequence structure, chromatin environment and evolutionary history (Eisenberg

55 and Levanon 2013; Rancati et al. 2018). For example, housekeeping genes are evolutionarily

56 ancient (Zhu et al. 2008), exhibit a high level of evolutionary conservation (Zhang and Li 2004),

57 and are enriched for several functions, including metabolism, RNA binding, protein degradation

58 and cytoskeleton functions (Zhang and Li 2004; Lehner and Fraser 2004).

59 While core functions are often described based on 'housekeeping genes', we recognize that

60 genes do not work in isolation, but work with each other to carry out biological processes.

61 Individual molecular abundances alone cannot adequately capture biological organizations. High-

62 throughput methods that generate high-dimensional 'omic' data have greatly increased our

63 understanding of molecular and cellular function and organization, in particular through the

64 analysis of molecular networks (Barabasi and Oltvai 2004; Proulx, Promislow, and Phillips 2005;

65 Thompson, Regev, and Roy 2015; Promislow 2005). Studying core functions from a network

66 perspective may provide novel insights into biological organization. Networks consist of nodes

67 connected to one another by edges. In the search for the underlying molecular structure of cells,

68 researchers have explored many different kinds of edges, including but not limited to gene co-

69 expression, protein-protein interactions (PPI), interactions among transcription factors (TF), TF

70 chromatin occupancy, miRNA-target gene interactions, metabolites covariation, and metabolic

71 reactions (Mitra et al. 2013). For example, co-expression network analysis of human and

72 Arabidopsis bulk transcriptome data has found a substantial number of gene pairs whose co-

73 expression spans multiple datasets (Lee et al. 2004; He and Maslov 2016). In both analyses, gene 
74 pairs expressed across samples were enriched in translation, DNA replication, and regulation of

75 transcription functions, all generally considered to be core cellular functions. Recent studies of

76 tissue-level transcriptome data have typically focused on tissue-specific networks (Greene et al.

77 2015; Sonawane et al. 2017). For example, Skinnider et al. (2021) constructed tissue-specific PPI

78 networks using co-immunoprecipitation within each of seven mouse tissues. They discovered core

79 cellular modules, present in all mouse tissues, composed of evolutionarily ancient proteins, which

80 contrasts with evolutionarily novel accessory modules that are found within individual tissues.

A major drawback of most previous studies is that the networks were inferred from bulk data,

82 which profiles heterogeneous cell populations of an organism or in a tissue. Bulk samples face two

83 main limitations for network construction. First, differences in cellular compositions between

84 samples may confound covariation analysis (Farahbod and Pavlidis 2020). Second, measurements

85 that are averaged over thousands of cells in bulk samples make it difficult to detect interactions

86 between genes in individual cells, such as the presence of co-expression patterns and the cell-

87 specificity of these interactions. Co-expression in particular is an indicator of functional

88 relationships (Hughes et al. 2000), which from a network perspective can provide valuable insight

89 into cell function (Barabasi and Oltvai 2004). The compendium of core housekeeping genes,

90 initially characterized based on the consistency of their expression, may change based on further

91 analyses of gene-gene relationships. For example, does each commonly expressed gene interact

92 with other genes in a fixed and static manner in all cell types, or do the interactions themselves,

93 which define the gene network structure, differ depending on the local cellular contexts? We can

94 gain a clearer understanding of core gene regulatory networks through the analysis of single-cell

95 sequencing data from a network perspective. 
With the advent of single-cell RNA sequencing (scRNA-seq), we have an unprecedented

97 opportunity to reveal gene relationships in specific cellular contexts and probe cellular-level

98 networks (Trapnell 2015; Tanay and Regev 2017). One recent study used single cell data from

99 mouse brain samples to construct gene co-expression networks and compared the topology of networks built from different cell type hierarchy levels (i.e., from broad to specific class, subclass,

101 and cluster labels of cell types; Harris et al. 2021). Their results show a high preservation of gene-

102 gene relationships at each hierarchy level and suggest the existence of a core co-regulatory network

103 in the brain. However, they did not directly compare cellular networks across cell types to find 104 commonality or describe a concrete core network with topological and functional features.

Taking together, the prior findings, and the considerable amount of scRNA-seq data now available, lead us to ask several fundamental questions: Can we identify shared co-expression

107 patterns between pairs of genes across different cell types, how common are these specific 108 connections across different cell types, do these shared co-expressed genes define a core cellular 109 network, and if so, what properties does this core network manifest?

To investigate these questions, we used a published scRNA-seq dataset derived from whole

111 fly brains (Davie et al. 2018) and constructed cell type-specific gene co-expression networks.

112 Furthermore, we described the functional enrichment of this network, and the evolutionary age of

113 its constituent genes. Gaining such information not only allows us to understand the composition

114 and function of the detected core network, but also provides insight into the molecular organization

115 of gene co-expression networks and the evolutionary origins of cellular functions. To our

116 knowledge, this is the first study searching for a core cellular network among cell types using

117 single cell data in the fly brain. 


\section{Results}

\section{Construction of cell type-specific gene co-expression networks}

We selected an array of fly brain cell types and filtered expressed genes before network construction. The original dataset contained 17,473 gene expression profiles in 56,902 high-quality brain cells grouped into 116 cell clusters. We selected 33 known cell types that contained at least 200 cells and filtered expressed genes in each cell type separately (Methods and Figure S1). Different cell types showed different numbers of expressed genes, ranging from a minimum of 3,153 expressed genes in the Tm9 cell type, to a maximum of 6,725 expressed genes in the ensheathing glia cell type (Figure 1). In total, there were 8,013 genes expressed in at least one cell type, 2,368 of which were expressed in all 33 cell types (Figure 1). Throughout, we focus on these 2,368 commonly expressed genes to identify covarying gene pairs within and across cell types. expressed gene pairs (21.4\% of all possible pairs) that occurred in at least one of the 33 cell type133 specific networks.

\section{Co-expression networks in fly brain cell types are highly context-dependent}

137 between two nodes exists if the genes are significantly correlated with each other across cells 138 within a specific cell type. If a core cellular network exists, we expect edges comprising this core 
139

140

141

142

143

144

145

146

147

148

149

to be present in all cell types. We define the number of cell types in which each edge (i.e., each co-expressed gene pair) is detected as that edge's 'commonality'. The distribution of commonality scores exhibited a monotonic decline over most of the range, with more than $75 \%$ of the edges specific to one cell type and only $0.5 \%$ of edges common to more than 10 cell types (Figure 2 ). The largest observed commonality score was 29 , and was observed for only one edge. The frequency of edge commonality initially decreased rapidly. However, this trend attenuated at approximately an edge commonality of 10 . The frequency of commonality stayed at a roughly constant level until 22, before it finally dropped to 0 above 29 (Figure 2). That is, among the 33 cell types we examined, no gene pairs were co-expressed in 30 or more cell types.

As a complement to the observed edge commonality distribution, we also plotted the gene commonality distribution, where gene commonality indicates the number of cell types in which a given gene was found to be significantly co-expressed with at least one other gene (i.e., to have at least one edge). The gene commonality distribution showed that most genes had one or more edges in the majority of cell types, and 176 genes had at least one edge in all 33 cell types (Figure 2). Thus, commonly expressed genes are frequently utilized and wired into co-expression circuits, though the specific wiring varies among different cell types.

\section{Recurrently co-expressed genes in multiple cell types}

We next asked to what extent the observed decline in edge commonality distribution with increasing cell types (Figure 2) differed from the null expectation. The null hypothesis provides the expected distribution of edge commonality when genes in each cell type are randomly coexpressed with each other, and we evaluate this in two ways. First, we derived a mathematical 
expectation for the probabilities of edge commonality using the binomial distribution (Methods).

162 For a gene pair to be co-expressed in 0,1 or 2 cell types, the probability values were $0.7177,0.2392$

163 or 0.0387 , respectively, indicating most gene pairs would be expected to co-express in no cells, or

164 only in one or two cell types. Given the 2,368 commonly expressed genes, we would expect to

165 find 791,068 unique gene pairs to occur (Methods). Our observation of only 600,888 such gene

166 pairs suggests that some genes recurrently co-express in multiple cell types. A full comparison of

167 this analytically predicted distribution and the observed edge commonality showed that the two

168 distributions agreed well at lower, more cell-specific commonality, but the discrepancy became

169 obvious for gene pairs found in $>3$ cell types (Figure S4). Second, we compared the deviation

170 between the observed edge commonality distribution and a null distribution using network

171 randomization (Methods). This comparison showed that the observed distribution was enriched

172 in high commonality edges. For instance, none of the randomizations generated an edge

173 commonality larger than 15, while the observed distribution included hundreds of edges with

174 commonality $\geq 15$ (Figure 3 ). This pattern is robust to the percentile cutoff values used in network

175 construction. When we applied a more stringent percentile cutoff and compared the observed with

176 null distributions, the discrepancy became even more prominent, shown as the increasing distance

177 between the two distributions measured using the Jensen-Shannon divergence (Figure S5). These

178 results suggest that there exists a set of co-varying genes that occur more repeatedly than expected

179 by chance across diverse cellular contexts.

In sum, despite the very large number of cell type-specific gene co-expression edges, our 181 analysis points to a core cellular network composed of genes that are co-expressed irrespective of 182 cellular contexts. 


\section{Topology pinpoints a core cellular network}

185

186

187

188

189

190

191

192

193

194

By definition, co-expressed genes with high commonality are more likely to contribute to a core cellular network. Although we searched for a core network shared by all cells, we did not observe any edges shared by all cell types. This discrepancy may be due to the percentile cutoff that we used in network construction. Indeed, networks based on a less stringent cutoff value of the top $10 \%$ of expressed genes contained edges common to all cell types, and the enrichment of high commonality edges remained (Figure S5). Less stringent cutoffs come with a higher risk of false positive co-expression edges, as indicated by the presence of high-commonality edges in the randomized networks at low stringency (Figure S5). Therefore, we continued our analysis with only the top $1 \%$ of edges, and leveraged other information to identify co-expressed genes that might reside in a core cellular network.

Previous network studies suggest that shared edges tend to be tightly connected with each other (Huttlin et al. 2021). We first evaluated the neighborhood density of high commonality edges and then measured the clustering coefficients of networks at different edge commonality cutoffs to determine an informative commonality cutoff value. We combined all cell type-specific networks into a pan-network whose edge weights reflected edge commonality and explored the relationship between edge commonality and edge clustering coefficient (Methods). To aid in comparison, we subsampled 10,000 edges for edge groups that contained $>10,000$ edges to make them more comparable to edge groups with $<10,000$ edges. The result showed that edge commonality and edge clustering coefficient were positively correlated (Figure 4A), supporting the idea that recurrently co-expressed genes tend to reside in dense subnetwork neighborhoods. 
Next, we progressively extracted edges with increasing commonality cutoffs and calculated each resulting subnetwork's clustering coefficient. The result showed that with cutoff values of

207 increasing stringency, the clustering coefficient increased to a peak of 0.75 at a cutoff value of 14 ,

208 declining thereafter (Figure 4B). Based on this result, we chose a commonality of 14 as the 209 minimum value for including an edge in a core cellular network, which gave 1810 non-redundant 210 edges among 179 genes (Figure 4C).

To evaluate the connectivity of the core network, we calculated its clustering coefficient and compared it to an ensemble of coefficients from pseudo core networks each with the same number 213 of genes, edges, and degree distribution as the observed one. The mean simulated clustering

214 coefficient value was 0.15 , with a range of 0.12 to 0.18 , much smaller than the observed value 0.75

215 (Figure S6).

We noticed that edges in the defined core network were not present in every surveyed cell type.

217 This could be due to our parameter value choices, such as correlation percentile cutoff or edge 218 commonality cutoff, or alternatively, these edges or gene pairs are not co-expressed in all cell types.

219 To examine these possibilities in detail, we looked at the rank of these edges' correlation among 220 the distribution of edge correlation values in cell types in which they were below the top $1 \%$. In 221 particular, did they show consistently high ranked correlations across these remaining cell types?

222 To quantify patterns over cell types, we used a rank aggregation method and estimated a $\mathrm{P}$ value 223 per edge (Method). The estimated $\mathrm{P}$ value ranges from 0 to 1 and serves as an upper bound of the 224 computationally expensive exact $\mathrm{P}$ value, with a small value indicating one edge is ranked 225 consistently higher across cell types and a larger value meaning one edge's rank distribution over 226 cell types follows a random pattern. Due to computational limitations, we randomly sampled 100 227 edges in 10 edge commonality groups separately and computed their respective P values (Figure 
228

229

230

231

232

233

234

235

236

237

238

239

240

241

242

243

244

245

246

247

248

249

S7). More than 94 percent of edges sampled from the core network (edge commonality $\geq 14$ ) are highly ranked in the remaining cell types $(\mathrm{P}<0.05)$, whereas none of the 100 cell type-specific edges (edge commonality $=1)$ that were tested were significant $(\mathrm{P}>0.05$ in all cases), suggesting that although these core edges did not make to the top $1 \%$ correlations in a set of cell types, they are relatively highly co-expressed across the remaining cell types.

\section{Structure, function and evolutionary signatures of the core cellular network}

Having defined a core cellular network, we next examined its structure, functional enrichment and evolutionary signature. Many complex networks can be divided into modules, where genes are more highly interconnected within modules than between modules (Newman 2003). Modules identified from gene co-expression networks tend to take part in the same biological processes or pathways (Ruprecht, Proost, et al. 2017; Wolfe, Kohane, and Butte 2005). To explore the structural and functional organization of this core, we decomposed it into highly connected modules using the Markov Clustering Algorithm (Methods). In total, we identified seven modules with at least five gene members (Figure 5 and Table S2). We then annotated each module's biological function through Gene Ontology (GO) enrichment analysis. The results revealed an array of 'housekeeping' and brain related functions enriched within different modules (Figure 5 and Table S3). The largest module (module 7) contained 78 genes, and was enriched for ribosome related functions, such as cytoplasmic translation, suggesting tight correlation of genes encoding ribosomal proteins across cells. The second largest module (module 6), contained 15 genes and was enriched for glycolysis,

a process central to cellular energy homeostasis. Modules 1 and 2 appeared to facilitate ATP metabolic process and proton transport, and module 3 was related to synaptic signaling, perhaps 
reflecting neuronal functions. Module 5 formed a fully interconnected subnetwork without any

252 players in protein folding. The smallest of the modules, module 4, showed enrichment in the

253 rhodopsin biosynthetic process.

254 To characterize the evolutionary signature of each module, we used phylostratigraphy, 255 assigning each gene in each module to one of 10 different evolutionary time periods (Table S4).

256 Broadly speaking, genes involved in the core network were enriched for genes with ancient origins,

257 compared with genes commonly expressed in the fly brain (Figure 6A). Looking in detail, 258 different modules had different gene age compositions (Figure 6B). Gene members of the 259 ribosomal (module 7) and protein folding (module 5) modules predated the divergence of the 260 eukaryota, while those of the ATP metabolic (module 1 and 2), glycolysis (module 6) and synaptic 261 signaling (module 3) modules included genes distributed across both ancient and relatively recent

262 evolutionary time periods. Module 4 showed the youngest age signature, with all gene members 263 emerging after eumetazoa. These diverse age signatures of different core modules suggest that 264 they arose by integration of both young and old genes, perhaps involving step-wise recruitment of 265 young genes into ancestral core modules.

267 Discussion

To what extent do all cells in an organism rely on a common core of interacting genes? To

269 investigate this question, we examined cell type-specific gene co-expression networks using fly

270 brain scRNA-seq data. We described a core gene co-expression network and found it to be small

271 and more densely connected relative to the larger, more cell-specific gene co-expression networks. 
272 The core is composed of numerous co-expression modules that appear distinct from each other in

273 terms of functional enrichment and the distribution of gene age.

274 Our study is distinct in at least three ways from previous work interrogating core networks.

275 First, a large body of studies has relied heavily on protein interactions to derive biological networks

276 and find commonalities. While these studies are informative, they suffer from the bias that protein

277 interaction data are enriched for highly-studied proteins, which may lead to an incomplete picture

278 of network structure (Skinnider, Stacey, and Foster 2018; Gillis, Ballouz, and Pavlidis 2014;

279 Schaefer, Serrano, and Andrade-Navarro 2015). Moreover, these studies are typically lacking

280 information on the cell specificity of such interactions. In our study, we analyzed transcriptome

281 data, which interrogate almost all genes in the genome and are less biased with respect to

282 knowledge from prior databases or existing literature.

283 Second, instead of relying on expression levels of individual genes to identify genes common

284 across cell types, we examined covariation between genes as the measure of functional

285 commonality, which provides not only a stricter criterion to infer gene function (Hughes et al.

286 2000), but also likely captures conserved gene regulatory networks (Yu et al. 2003; Stuart et al.

287 2003; Segal et al. 2003).

Third, we identified covarying gene pairs using scRNA-seq data, which unlike bulk tissue data,

289 can be defined by cell type, even within a single biological sample. In contrast to bulk 290 transcriptomic analysis and PPI data, where the cellular specificity of each interaction is largely

291 ambiguous, scRNA-seq enabled us to build cell type-specific networks at a resolution that has

292 hitherto not been possible. 


\section{Is there a core network active in all cells?}

295 To determine whether there is a core network common to all cells at the level of single-cell genegene covariation, we focused our analysis on genes that were expressed across all cell types, which

297 allowed us to directly compare the diverse co-expression patterns among a common set of genes.

298 We limit the question to the fly brain, acknowledging that the core modules we identify might be

299 absent in non-brain cells. Even among the genes expressed in all cell types, we found that the co-

300 expression of most genes was cell type-specific, and yet there also existed a significantly large 301 number of genes whose co-expression occurred among multiple cell types. This enrichment of

302

303

304

305

306

307

308

309

310

311

312

313

314

315

316

shared co-expressed genes suggests the existence of a core co-expression network across diverse cell types. We applied a relatively high statistical significance threshold to extract shared coexpressed genes, which we defined as the core network.

While we identified edges common to many cell types, our inferred core cellular network lacks edges shared by all cell types. We consider two alternative explanations for this observation. First, it is possible that a common core co-expression network for all brain cells does not exist, and that cell co-expression networks are so diverse as to lack such rigid network structure across the cells of the Drosophila brain. Alternatively, we considered the possibility that a core does exist, but that the statistical inference of gene co-expression and the threshold value we use to build the network might not fully resolve the core in all cells. To define the core, we specify multiple parameters, including a gene correlation metric, a correlation threshold to select co-expressed genes, and an edge commonality cutoff to extract a core network. Given that our definition of gene interactions is based on statistical inference of correlations, we might simply fail to observe a real interaction in one or more cell types due to type II error (false negatives). As we explored this parameter space, moving from relaxed to stringent parameter values, a few features of the network became apparent. 
317 In particular, more relaxed parameter values revealed co-expressed gene pairs observed in all cell

318 types in the brain, but this inherently increased the risk of false positive gene pairs (type I error),

319 as revealed by network permutation. In an effort to reduce the risk of false positives, we examined

320 a more conservative parameter space, which still yielded many edges shared widely across cell

321 types, but did not identify edges shared by all cells. We looked in detail at those edges that were

322 in our defined core cellular network, with edges present in $\geq 14$ cell types, and yet were absent

323 from the top $1 \%$ of most significantly correlated gene pairs in the remaining cell types. We found

324 that almost all of these edges were in fact ranked among the most highly co-expressed, but below

325 the initial threshold, in the remaining cell types. Thus, their absence from the core appeared to be

326 due to their relatively weak co-expression strengths compared to the cell type-specific gene pairs.

327 Together, our analysis indicates the existence of a core cellular network, though the size and

328 composition that we define is conditional on parameter choices.

\section{Topological properties of the core network}

With our current parameter choices, the defined core network is remarkably small when compared to the much larger network of cell-specific interactions. In particular, only $0.5 \%$ of coexpressed gene pairs are shared by $\geq 10$ cell types in the fly brain. This number is at the lower end

334 compared with previous studies of different biological networks. For example, Skinnider et al. 335 (2021) constructed tissue-specific PPI networks for seven mouse tissues and found $0.7 \%$ of all

336 detected PPIs were shared by all tissues. Neph et al. (2012) built TF interaction networks for 41

337 cell types in humans and found that five percent of interacting TFs were common to all cell types.

338 Almass et al. (2005) used flux-balance analysis to study active metabolic reactions of Escherichia 
coli in 30,000 diverse simulated environments and found 90 of $758(11.9 \%)$ reactions were always active.

What are the factors that contribute to the size of a core network? From a statistical perspective,

342 the number of co-expressed genes change along with parameter choices, which in turn modify the

343 observed core network size. From a biological perspective, different biological contexts, such as

344 organ, sex, genotype and age, might have condition-specific co-expressed gene pairs, which would

345 affect the core network edge compositions and hence its size. Our defined core network was

346 discovered using fly brains, a complex organ with highly heterogeneous cell type composition.

347 Projecting such an analysis to more organs, or even to a whole fly, would very likely reveal an

348 even smaller core, as the inclusion of a larger set of diverse cells would lead to a smaller set of

349 universal edges. Furthermore, some of the modules that we find in the core we describe here appear

350 to be related to the brain in particular, and so it seems reasonable to presume that those modules

351 would not be found in an analysis that includes more organs or tissues. We therefore speculate that

352 the relative core size of $0.5 \%$ that we observe is an overestimate of the true core network size for

353 all cells found in Drosophila melanogaster. While the 1810 edges and 179 genes in the core might

354 decrease in an organism-wide analysis, we postulate that such an analysis could ultimately identify

355 modules with a low but constant number of co-expressed genes due to the presence of a small core

356 network common to all cells in an individual. architecture we observed, which embodies extensive cell-type specific interactions along with a shared and densely connected core, echoes findings from other types of biological networks. For example, Liu et al. (2020) identified 13,764 PPIs in yeast across nine environments and found that $36160 \%$ of PPIs were found in only one environment. They also show that PPIs, present in $\geq 8$ 
environments, form 'tight' modules of high node degree, while PPIs in $\leq 3$ environments form lessconnected modules of smaller node degree (Liu et al. 2020). Protein interaction networks based on

364 just two human cell lines revealed that shared interactions tend to reside in dense subnetworks and 365 correspond to known protein complexes such as the exosome and the COP9 signalosome (Huttlin et al. 2021). Similarly, network analyses of gene co-expression from bulk transcriptomics in

367 Arabidopsis and humans suggest a highly connected core, which appears alongside an extensive 368 number of condition-specific gene interactions (He and Maslov 2016; Lee et al. 2004). Taken 369 together, these results suggest a universal organizing principle in biological systems, where widely 370 shared components of interaction networks are relatively small and densely connected (Milo et al. $3712004 ; 2002$; Csermely et al. 2013).

373 An evolutionary perspective of the multi-part core network

Previous work suggests that biological systems evolve and function in a modular fashion, 375 where groups of genes that share functional relationships tend to co-evolve independently of genes 376 in other functional groups, and where genes and proteins that share function tend to be co-regulated

377 (Hartwell et al. 1999; Schlosser and Wagner 2004; Ryan et al. 2012; Ge et al. 2001; Martin and 378 Fraser 2018). Consistent with this, we find the core network is modularly structured. Modules 379 within the core network have different evolutionary signatures and enrich different biological 380 functions. In our analysis of the age distributions of module gene members, we found some 381 modules with genes of ancient origin, and others with a mixture of ancient and young genes, and 382 at least one having a surprisingly young signature. 
It is obviously of great interest to determine the origination time and evolutionary dynamics of

384 the core network modules. As a first step, we dissected the module gene members into

385 phylostratographic age groups. However, this gene age information is not sufficient to infer

386 module ages, as the mere presence of two ancient genes is not equivalent to ancient co-expression,

387 genes can be co-opted to generate new functions by changing their patterns of regulation (Ruprecht,

388 Vaid, et al. 2017; Thompson, Regev, and Roy 2015). To better estimate the evolutionary origin of

389 each module, we would need to apply a phylogenetic analysis of gene co-expression, and thus

390 module dynamics, across multiple species. Given the focus here on a single species, we cannot

391 infer the degree of evolutionary conservation of the co-expression relationships themselves, and

392 so we cannot determine if the network modules we describe are themselves of different ages. That

393 said, there is still some evolutionary inference we can draw based on the evolutionary ages of the

394 genes within each module. Gene modules with a young age signature are only feasible after those

395 young genes emerged. Thus, gene age sets an upper bound on module origination time. For

396 example, the ages of genes in module 4 are distributed among 'Bilateria', 'Protostomia', and

397 'Arthropoda' evolutionary periods. The most recent of these groups, the Arthropoda, thus sets an

398 upper bound on module age - it must have arisen sometime after the origin of arthropods.

Our observation of core modules with genes of different ages is in line with previous

400 comparative studies based on bulk transcriptomics, which suggest that core modules may have

401 evolved at different times (Pembroke, Hartl, and Geschwind 2021; Stuart et al. 2003). Our work

402 however points toward a more limited core network, both in size and in function, than these studies.

403 At the resolution afforded by bulk transcriptomic analysis, ancient co-expression modules

404 preserved across species appear quite broad in function, including ribosome, proteasome, energy

405 generation, cell cycle, secretion, transcription and translation functions (Stuart et al. 2003), while 
406 less evolutionarily conserved modules are naturally involved in an even broader range of functions

407 (Pembroke, Hartl, and Geschwind 2021; Stuart et al. 2003). We show however that even in a single

408 species, the conserved core network is enriched in a smaller range of functions, and we argue that

409 this is an overestimate of the core network that may be conserved across a whole organism, much

410 less across species. Of the two modules that we describe that consist of evolutionarily ancient

411 genes, one was functionally enriched for ribosome biogenesis and the other for protein folding.

412 Due to the gene-age distribution of these modules relative to the others we identify, we speculate

413 that these two modules are most likely to be conserved across the widest range of cells and of

414 species. The 'hybrid' modules, containing both young and old genes, enrich ATP metabolic

415 processes, oxidative phosphorylation, glycolysis, and chemical synaptic transmission. This pattern

416 points to these hybrid core modules being important for energy metabolism, perhaps in brain cells

417 in particular, but regardless, we suspect that these modules would be less conserved across cells

418 and species. To obtain a more complete understanding of the emergence, assembly and 419 evolutionary dynamics of the core cellular network and its conserved functions, future studies 420 should focus on obtaining single cell data from more tissues and from multiple species.

\section{Limitation and future directions}

423 In this study, we sought a core of interacting genes found across cell types in the fly brain.

424 While the work described here benefits from access to high quality single-cell transcriptome data,

425 there are still several caveats worth noting. First, the fly brain cell atlas (Davie et al. 2018) was

426 generated using a mixture of two genotypes, and with cells from both female and male fly brains

427 and from individuals of several ages. Thus, genotype, sex or age-specific gene co-expression 
428 patterns are hidden. Future studies targeting individual genotypes, separate sexes, and/or specific

429 age groups might identify core networks that differ by age, sex or genotype. Second, we inferred

430 co-expressed gene pairs from gene expression data statistically. Gene co-expression is not

431 equivalent to gene co-regulation, which may be more indicative of functional relationships. Further

432 experimental work is needed to validate the functional implications of these gene pairs. Lastly, we

433 note that this analysis is based on scRNA-seq data, rather than single nuclear RNA-seq. These two

434 approaches might capture different aspects of cell activity (H. Wu et al. 2019; Denisenko et al.

435 2020; Thrupp et al. 2020).

To fully understand the molecular basis of cell functions, we need to integrate biological

437 networks from different domains - e.g., transcriptome, proteome, or metabolome. It would be of

438 considerable interest to compare core networks defined for each domain and to study their

439 relationships. For example, it has been shown that genes whose protein products physically interact

440 tend to be co-expressed together, suggesting an interdependence of network structure between

441 biological domains (Fraser et al. 2004; Ge et al. 2001; Lemos, Meiklejohn, and Hartl 2004). A

442 number of challenges exist however, such as how to define core networks for different types of

443 data, and how to relate networks between domains (Civelek and Lusis 2014; Mitra et al. 2013).

444 Future studies addressing these challenges may identify a systematic approach to comprehensively

445 survey and compare core networks in different domains. Such an analysis could elucidate the 446 organizing principles of cellular networks and provide deeper insight into the evolutionary origin

447 and molecular functions shared by all cells. 


\section{Conclusions}

450 In summary, we leveraged single cell transcriptome data to reveal a core cellular network in fly

451 brains, and deciphered its topological, structural, functional, and evolutionary properties. Our

452 study demonstrates that studying single cell data through a network approach can provide novel

453 insights into understanding cellular functions in a complex organ. It would be valuable to apply

454 our analysis to similar data from different organs, and in multiple species, to reveal the most 455 conserved network components, and the core networks that exist in cells of all multicellular 456 organisms.

$458 \quad$ Materials and Methods

\section{Dataset collection and preprocessing}

We downloaded the fly brain atlas data from NCBI Gene Expression Omnibus with GEO accession 'GSE107451'. The original dataset contains 17,473 gene expression data in 56,902 highquality brain cells grouped into 115 cell clusters. As a quality control step, we first removed 668

463 cells in a cell cluster named 'Hsp' as they represent stressed cells (Jasper Janssens, personal

464 communication). We then removed cells that had either less than 200 expressed genes, less than 465500 total unique molecular identifier counts, or a total fraction of mitochondrial gene expression 466 exceeding 30\%. These criteria led to the removal of another 42 cells, leaving 56,192 cells. These 467 cells were annotated to 115 cell clusters (Davie et al. 2018). We selected 33 cell clusters which 468 had at least 200 cells and were annotated to known brain cell types in the following analyses 469 (Figure S1). 
470 We filtered genes for each cell type individually by removing genes that were expressed in less

471 than 15 cells, or in fewer than $0.5 \%$ of cells in that cell type. This gene filtering procedure led to

4728013 genes as expressed in at least one cell type, 2368 of which were commonly expressed in all

47333 cell types (Figure $\mathbf{S 1})$.

474

475 Constructing cell type-specific gene co-expression networks

476 We used the bigScale2 algorithm (Iacono, Massoni-Badosa, and Heyn 2019) to compute a

477 gene-gene correlation matrix for each cell type (Figure S2). This algorithm was tailored to mitigate

478 the impact of sparse counts at the single-cell level. It first groups cells into homogenous cell

479 clusters, then performs differential expression (DE) analysis between all pairs of clusters. With $\mathrm{N}$

480 clusters, we obtain $\mathrm{N}^{*}(\mathrm{~N}-1) / 2$ unique comparisons and each comparison generates one Z-score for

481 each gene, indicating the likelihood of an expression change between the corresponding two

482 clusters. Finally, bigScale2 uses transformed Z-scores instead of original expression values to

483 calculate Pearson correlation coefficients (Figures S3). For each cell type, we ranked gene pairs

484 by their absolute correlation values and placed the top $1 \%$ of correlated gene pairs into a co-

485 expression network, with the corresponding absolute correlation values ranging from 0.53 to 0.93 .

486 Network summary statistics, including major component size, average path length, and clustering

487 coefficient were calculated using functions from the R package iGraph (Csardi and Nepusz 2006)

488 (Table S1). 


\section{Computing gene and edge commonality distributions}

To evaluate commonality and specificity across cell type-specific networks, we plot the node and edge commonality distributions. The commonality of a node (gene) refers to the number of

493 cell types in which this gene was found to be co-expressed (edge) with at least one other gene. The 494 commonality of an edge linking a given pair of genes refers to the number of cell types in which 495 this edge is detected.

\section{A mathematical approximation of the edge commonality distribution} probability $P(k)$ of a gene pair co-expressed in $k$ cell types equal

$$
P(k)=C(33, k) * 0.01^{k} *(1-0.01)^{33-k}
$$

504 with the first term representing the combinatorial number describing the number of ways of

505 picking $k$ items from a pool of 33 cell types. Following this equation, the probability of a gene pair 506 not co-expressed in any cell type is 0.7177 at $\mathrm{k}=0$. Given the 2,368 commonly expressed genes,

$507 C(2358,2) *(1-0.7177)=791,068$ non-redundant gene pairs were expected to co-express in at 508 least one cell type. 


\section{Network randomization}

To obtain a null distribution for edge commonality distributions, we used a network

512 randomization approach. We randomized the edges in each cell type-specific network individually

513 keeping the gene connectivities fixed using the rewire function in iGraph. A set of randomizations

514 for all 33 cell types resulted in one pseudo edge commonality distribution. We performed the

515 randomization procedure 100 times and used the ensemble of the 100 pseudo edge commonality

516 distributions as the null distribution. We computed the null distribution with different percentile

517 cutoff values and used Jensen-Shannon divergence with the JSD function from the R package

518 Philentropy (Drost 2018) to measure the distance between two distributions.

520 Calculation of clustering coefficient per edge

To determine the topological properties of edges with different levels of commonality, we

522 calculated a clustering coefficient for each edge following the approach in Huttlin et al. 2021. We

523 combined all cell type-specific networks into a pan-network whose edge weights reflected edge

524 commonality. For each edge in the pan-network, we extracted all first-degree neighbors of the two

525 genes constituting this edge. These neighbors and the two focal genes defined a subgraph from the

526 whole pan-network. For each subgraph, we calculated the clustering coefficient as the number of

527 triangles (3 vertices with three edges) divided by the number of connected triples (3 vertices with

528 two edges). As the clustering coefficient of a graph is related to its connectivity, or edge density,

529 a high local clustering coefficient indicates that this edge is located in a dense subnetwork. 


\section{$531 \quad$ Rank aggregation analysis}

Each gene pair or edge has an rank based on its absolute correlation value in a given cell type.

533 To quantify if one edge is ranked consistently higher across a set of cell types based on its absolute

534 correlation value, we used the aggregateRanks function from the R package RobustRankAggreg

535 (Kolde et al. 2012). This function is based on a probabilistic model of order statistics and computes

536 a derived $\mathrm{P}$ value for each edge. The derived $\mathrm{P}$ value ranges from 0 to 1 and serves as an upper

537 bound of the computationally expensive exact $\mathrm{P}$ value, with a small value indicating one edge is

538 ranked consistently higher across cell types and a larger value meaning one edge's rank

539 distribution over cell types follows a random pattern. We chose 10 edge commonality groups: 1,

$5404,8,10,12,14,16,18,20$, and 22 . Within each edge commonality group, we randomly sampled

541100 edges. For each edge, we first collected the cell types that this edge was absent (didn't make

542 to the top $0.1 \%$ correlations), and then calculated the derived $\mathrm{P}$ values on these cell types. The

543 derived $\mathrm{P}$ values were corrected for multiple testing using the p.adjust function in $\mathrm{R}$ with the

544 Benjamini-Hochberg method, referred to as adjusted $\mathrm{P}$ values hereafter. We plotted the adjusted $\mathrm{P}$

545 value distribution of the 100 sampled edges for each edge commonality group separately.

547 Module decomposition and functional annotation of the core

548 To decompose the core cellular network into highly connected modules, we used the mcl

549 function from the R package MCL (Jäger 2015) which implements a Markov cluster algorithm to

550 identify clusters in networks. After module detection, we performed Gene Ontology (GO)

551 enrichment analysis of genes in each module using the R package clusterProfiler (T. Wu et al.

552 2021) with a Bonferroni correction and an adjusted $P$ value cutoff of 0.05. Significant GO terms 
553 were identified and refined to reduce redundant GO terms via the simplify method from the

554 clusterProfiler package.

556 Assigning genes into evolutionary age groups

557 We downloaded data from a previous study to assign genes into different evolutionary age

558 groups using a phylostratigraphy framework (Domazet-Lošo et al. 2017). This framework allows

559 us to date the evolutionary origination time of a gene by identifying its homologs across the tree

560 of life. There were 13,794 genes assigned to 12 age groups in the original publication, 2,222 of

561 which overlapped with the 2,368 expressed genes in this study, including 1,021 genes in the oldest

562 age group "CellLife", 707 in "Eukaryota", 88 in "Opisthokonta", 140 in "Metazoa", 34 in

563 "Eumetazoa", 78 in "Bilateria", 15 in "Protostomia", 17 in "Arthropoda", 16 in "Pancrustacea",

56446 in "Insecta", 48 in "Diptera" and 12 in the youngest age group "Drosophila".

566 Declarations

567 Ethics approval and consent to participate

568 Not applicable

569 Consent for publication

570 Not applicable 


\section{Availability of data and materials}

572 The fly brain atlas dataset analyzed during the current study is available at GEO with accession

573 number GSE107451. R scripts for data analyses are available from the following GitHub

574 repository: https://github.com/mingwhy/fly.brain.core_coexpr.net.

\section{Competing interests}

576 The authors declare that they have no competing interests.

\section{$577 \quad$ Funding}

578 This work was supported in part by National Institute on Aging grants R21AG56872901, 579 R01AG057330, and R01AG063371.

\section{Authors' contributions}

581 MY and DP designed the study. MY collected and analyzed the data. MY, BH and DP interpreted

582 the data and wrote the manuscript. All authors read and approved the final manuscript.

\section{Acknowledgements}

584 This work was in part facilitated through the use of advanced computational, storage, and

585 networking infrastructure provided by the Hyak supercomputer system at the University of 586 Washington. 


\section{References}

589

590

591

592

593

594

595

596

597

598

599

600

601

602

603

604

605

606

607

608

609

610

611

612

613

614

615

Almaas, Eivind, Zoltán N Oltvai, and Albert-László Barabási. 2005. "The Activity Reaction Core and Plasticity of Metabolic Networks.” PLoS Computational Biology 1 (7): e68.

Barabasi, Albert-Laszlo, and Zoltan N Oltvai. 2004. "Network Biology: Understanding the Cell's Functional Organization." Nature Reviews Genetics 5 (2): 101-13.

Civelek, Mete, and Aldons J Lusis. 2014. "Systems Genetics Approaches to Understand Complex Traits." Nature Reviews Genetics 15 (1): 34-48.

Csardi, Gabor, and Tamas Nepusz. 2006. "The Igraph Software Package for Complex Network Research.” InterJournal, Complex Systems 1695 (5): 1-9.

Csermely, Peter, András London, Ling-Yun Wu, and Brian Uzzi. 2013. "Structure and Dynamics of Core/Periphery Networks.” Journal of Complex Networks 1 (2): 93-123.

Davie, Kristofer, Jasper Janssens, Duygu Koldere, Maxime De Waegeneer, Uli Pech, Łukasz Kreft, Sara Aibar, Samira Makhzami, Valerie Christiaens, and Carmen Bravo GonzálezBlas. 2018. "A Single-Cell Transcriptome Atlas of the Aging Drosophila Brain." Cell 174 (4): $982-98$.

Denisenko, Elena, Belinda B Guo, Matthew Jones, Rui Hou, Leanne De Kock, Timo Lassmann, Daniel Poppe, Olivier Clément, Rebecca K Simmons, and Ryan Lister. 2020. "Systematic Assessment of Tissue Dissociation and Storage Biases in Single-Cell and Single-Nucleus RNA-Seq Workflows.” Genome Biology 21: 1-25.

Domazet-Lošo, Tomislav, Anne-Ruxandra Carvunis, Mar Albà, Martin Sebastijan Šestak, Robert Bakarić, Rafik Neme, and Diethard Tautz. 2017. "No Evidence for Phylostratigraphic Bias Impacting Inferences on Patterns of Gene Emergence and Evolution." Molecular Biology and Evolution 34 (4): 843-56.

Drost, Hajk-Georg. 2018. "Philentropy: Information Theory and Distance Quantification with R." Journal of Open Source Software 3 (26): 765.

Eisenberg, Eli, and Erez Y Levanon. 2013. "Human Housekeeping Genes, Revisited." Trends in Genetics 29 (10): 569-74.

Farahbod, Marjan, and Paul Pavlidis. 2020. "Untangling the Effects of Cellular Composition on Coexpression Analysis." Genome Research 30 (6): 849-59.

Fraser, Hunter B, Aaron E Hirsh, Dennis P Wall, and Michael B Eisen. 2004. "Coevolution of Gene Expression among Interacting Proteins." Proceedings of the National Academy of Sciences 101 (24): 9033-38. 
Ge, Hui, Zhihua Liu, George M Church, and Marc Vidal. 2001. "Correlation between Transcriptome and Interactome Mapping Data from Saccharomyces Cerevisiae.” Nature Genetics 29 (4): 482-86.

Ghadie, Mohamed A, Jasmin Coulombe-Huntington, and Yu Xia. 2018. "Interactome Evolution: Insights from Genome-Wide Analyses of Protein-Protein Interactions." Current Opinion in Structural Biology 50: 42-48.

Gillis, Jesse, Sara Ballouz, and Paul Pavlidis. 2014. "Bias Tradeoffs in the Creation and Analysis of Protein-Protein Interaction Networks." Journal of Proteomics 100: 44-54.

Greene, Casey S, Arjun Krishnan, Aaron K Wong, Emanuela Ricciotti, Rene A Zelaya, Daniel S Himmelstein, Ran Zhang, Boris M Hartmann, Elena Zaslavsky, and Stuart C Sealfon. 2015. "Understanding Multicellular Function and Disease with Human Tissue-Specific Networks." Nature Genetics 47 (6): 569-76.

Harris, Benjamin D, Megan Crow, Stephan Fischer, and Jesse Gillis. 2021. "Single-Cell CoExpression Analysis Reveals That Transcriptional Modules Are Shared across Cell Types in the Brain." Cell Systems.

Hart, Yuval, and Uri Alon. 2013. "The Utility of Paradoxical Components in Biological Circuits." Molecular Cell 49 (2): 213-21.

Hartwell, Leland H, John J Hopfield, Stanislas Leibler, and Andrew W Murray. 1999. "From Molecular to Modular Cell Biology.” Nature 402 (6761): C47-52.

He, Fei, and Sergei Maslov. 2016. "Pan-and Core-Network Analysis of Co-Expression Genes in a Model Plant.” Scientific Reports 6 (1): 1-11.

Hughes, Timothy R, Matthew J Marton, Allan R Jones, Christopher J Roberts, Roland Stoughton, Christopher D Armour, Holly A Bennett, Ernest Coffey, Hongyue Dai, and Yudong D He. 2000. "Functional Discovery via a Compendium of Expression Profiles." Cell 102 (1): 109-26.

Huttlin, Edward L, Raphael J Bruckner, Jose Navarrete-Perea, Joe R Cannon, Kurt Baltier, Fana Gebreab, Melanie P Gygi, Alexandra Thornock, Gabriela Zarraga, and Stanley Tam. 2021. "Dual Proteome-Scale Networks Reveal Cell-Specific Remodeling of the Human Interactome." Cell 184 (11): 3022-40.

Iacono, Giovanni, Ramon Massoni-Badosa, and Holger Heyn. 2019. "Single-Cell Transcriptomics Unveils Gene Regulatory Network Plasticity.” Genome Biology 20 (1): 120.

Jäger, Martin L. 2015. "MCL: Markov Cluster Algorithm. R Package Version 1.0.” Https://CRAN.R-Project.Org/Package=MCL.

Kolde, Raivo, Sven Laur, Priit Adler, and Jaak Vilo. 2012. "Robust Rank Aggregation for Gene List Integration and Meta-Analysis." Bioinformatics 28 (4): 573-80. 
Lee, Homin K, Amy K Hsu, Jon Sajdak, Jie Qin, and Paul Pavlidis. 2004. "Coexpression Analysis of Human Genes across Many Microarray Data Sets." Genome Research 14 (6): 1085-94.

Lehner, Ben, and Andrew G Fraser. 2004. "Protein Domains Enriched in Mammalian TissueSpecific or Widely Expressed Genes." Trends in Genetics 20 (10): 468-72.

Lemos, Bernardo, Colin D Meiklejohn, and Daniel L Hartl. 2004. "Regulatory Evolution across the Protein Interaction Network.” Nature Genetics 36 (10): 1059-60.

Lim, Wendell A, Connie M Lee, and Chao Tang. 2013. "Design Principles of Regulatory Networks: Searching for the Molecular Algorithms of the Cell." Molecular Cell 49 (2): 202-12.

Liu, Zhimin, Darach Miller, Fangfei Li, Xianan Liu, and Sasha F Levy. 2020. "A Large Accessory Protein Interactome Is Rewired across Environments.” Elife 9: e62365.

Martin, Trevor, and Hunter B Fraser. 2018. "Comparative Expression Profiling Reveals Widespread Coordinated Evolution of Gene Expression across Eukaryotes." Nature Communications 9 (1): 1-9.

Milo, Ron, Shalev Itzkovitz, Nadav Kashtan, Reuven Levitt, Shai Shen-Orr, Inbal Ayzenshtat, Michal Sheffer, and Uri Alon. 2004. "Superfamilies of Evolved and Designed Networks." Science 303 (5663): 1538-42.

Milo, Ron, Shai Shen-Orr, Shalev Itzkovitz, Nadav Kashtan, Dmitri Chklovskii, and Uri Alon. 2002. "Network Motifs: Simple Building Blocks of Complex Networks." Science 298 (5594): 824-27.

Mitra, Koyel, Anne-Ruxandra Carvunis, Sanath Kumar Ramesh, and Trey Ideker. 2013. "Integrative Approaches for Finding Modular Structure in Biological Networks." Nature Reviews Genetics 14 (10): 719-32.

Neph, Shane, Andrew B Stergachis, Alex Reynolds, Richard Sandstrom, Elhanan Borenstein, and John A Stamatoyannopoulos. 2012. "Circuitry and Dynamics of Human Transcription Factor Regulatory Networks.” Cell 150 (6): 1274-86.

Newman, Mark E J. 2003. "The Structure and Function of Complex Networks." SIAM Review 45 (2): 167-256.

Parfrey, Laura Wegener, and Daniel J G Lahr. 2013. "Multicellularity Arose Several Times in the Evolution of Eukaryotes (Response to DOI 10.1002/Bies. 201100187).” Bioessays 35 (4): $339-47$.

Pembroke, William G, Christopher L Hartl, and Daniel H Geschwind. 2021. "Evolutionary Conservation and Divergence of the Human Brain Transcriptome." Genome Biology 22 (1): $1-33$. 
Promislow, Daniel. 2005. "A Regulatory Network Analysis of Phenotypic Plasticity in Yeast." The American Naturalist 165 (5): 515-23.

Proulx, Stephen R, Daniel E L Promislow, and Patrick C Phillips. 2005. "Network Thinking in Ecology and Evolution." Trends in Ecology \& Evolution 20 (6): 345-53.

Rancati, Giulia, Jason Moffat, Athanasios Typas, and Norman Pavelka. 2018. "Emerging and Evolving Concepts in Gene Essentiality." Nature Reviews Genetics 19 (1): 34-49.

Ruprecht, Colin, Sebastian Proost, Marcela Hernandez-Coronado, Carlos Ortiz-Ramirez, Daniel Lang, Stefan A Rensing, Jörg D Becker, Klaas Vandepoele, and Marek Mutwil. 2017. "Phylogenomic Analysis of Gene Co-expression Networks Reveals the Evolution of Functional Modules." The Plant Journal 90 (3): 447-65.

Ruprecht, Colin, Neha Vaid, Sebastian Proost, Staffan Persson, and Marek Mutwil. 2017. "Beyond Genomics: Studying Evolution with Gene Coexpression Networks." Trends in Plant Science 22 (4): 298-307.

Ryan, Colm J, Assen Roguev, Kristin Patrick, Jiewei Xu, Harlizawati Jahari, Zongtian Tong, Pedro Beltrao, Michael Shales, Hong Qu, and Sean R Collins. 2012. "Hierarchical Modularity and the Evolution of Genetic Interactomes across Species." Molecular Cell 46 (5): 691-704.

Schaefer, Martin H, Luis Serrano, and Miguel A Andrade-Navarro. 2015. "Correcting for the Study Bias Associated with Protein-Protein Interaction Measurements Reveals Differences between Protein Degree Distributions from Different Cancer Types." Frontiers in Genetics 6: 260 .

Schlosser, Gerhard, and Günter P Wagner. 2004. Modularity in Development and Evolution. University of Chicago Press.

Segal, Eran, Michael Shapira, Aviv Regev, Dana Pe'er, David Botstein, Daphne Koller, and Nir Friedman. 2003. "Module Networks: Identifying Regulatory Modules and Their ConditionSpecific Regulators from Gene Expression Data.” Nature Genetics 34 (2): 166-76.

Skinnider, Michael A, Nichollas E Scott, Anna Prudova, Craig H Kerr, Nikolay Stoynov, R Greg Stacey, Queenie W T Chan, David Rattray, Jörg Gsponer, and Leonard J Foster. 2021. “An Atlas of Protein-Protein Interactions across Mouse Tissues.” Cell 184 (15): 4073-89.

Skinnider, Michael A, R Greg Stacey, and Leonard J Foster. 2018. "Genomic Data Integration Systematically Biases Interactome Mapping.” PLoS Computational Biology 14 (10): e1006474.

Smith, John Maynard, and Eors Szathmary. 1997. The Major Transitions in Evolution. Oxford University Press.

Sonawane, Abhijeet Rajendra, John Platig, Maud Fagny, Cho-Yi Chen, Joseph Nathaniel Paulson, Camila Miranda Lopes-Ramos, Dawn Lisa DeMeo, John Quackenbush, Kimberly 
Glass, and Marieke Lydia Kuijjer. 2017. "Understanding Tissue-Specific Gene Regulation." Cell Reports 21 (4): 1077-88.

Sorrells, Trevor R, and Alexander D Johnson. 2015. "Making Sense of Transcription Networks." Cell 161 (4): 714-23.

Stuart, Joshua M, Eran Segal, Daphne Koller, and Stuart K Kim. 2003. “A Gene-Coexpression Network for Global Discovery of Conserved Genetic Modules.” Science 302 (5643): 249 55.

Tanay, Amos, and Aviv Regev. 2017. "Scaling Single-Cell Genomics from Phenomenology to Mechanism.” Nature 541 (7637): 331-38.

Thompson, Dawn, Aviv Regev, and Sushmita Roy. 2015. "Comparative Analysis of Gene Regulatory Networks: From Network Reconstruction to Evolution.” Annual Review of Cell and Developmental Biology 31: 399-428.

Thrupp, Nicola, Carlo Sala Frigerio, Leen Wolfs, Nathan G Skene, Nicola Fattorelli, Suresh Poovathingal, Yannick Fourne, Paul M Matthews, Tom Theys, and Renzo Mancuso. 2020. "Single-Nucleus RNA-Seq Is Not Suitable for Detection of Microglial Activation Genes in Humans." Cell Reports 32 (13): 108189.

Trapnell, Cole. 2015. "Defining Cell Types and States with Single-Cell Genomics." Genome Research 25 (10): 1491-98.

Wagner, Andreas. 2012. "Metabolic Networks and Their Evolution.” In Evolutionary Systems Biology, 29-52. Springer.

Wolfe, Cecily J, Isaac S Kohane, and Atul J Butte. 2005. "Systematic Survey Reveals General Applicability of" Guilt-by-Association" within Gene Coexpression Networks." BMC Bioinformatics 6 (1): 1-10.

Wu, Haojia, Yuhei Kirita, Erinn L Donnelly, and Benjamin D Humphreys. 2019. “Advantages of Single-Nucleus over Single-Cell RNA Sequencing of Adult Kidney: Rare Cell Types and Novel Cell States Revealed in Fibrosis." Journal of the American Society of Nephrology 30 (1): 23-32.

Wu, Tianzhi, Erqiang Hu, Shuangbin Xu, Meijun Chen, Pingfan Guo, Zehan Dai, Tingze Feng, Lang Zhou, Wenli Tang, and Li Zhan. 2021. "ClusterProfiler 4.0: A Universal Enrichment Tool for Interpreting Omics Data.” The Innovation 2 (3): 100141.

Yu, Haiyuan, Nicholas M Luscombe, Jiang Qian, and Mark Gerstein. 2003. “Genomic Analysis of Gene Expression Relationships in Transcriptional Regulatory Networks." Trends in Genetics 19 (8): 422-27.

Zhang, Liqing, and Wen-Hsiung Li. 2004. "Mammalian Housekeeping Genes Evolve More Slowly than Tissue-Specific Genes.” Molecular Biology and Evolution 21 (2): 236-39. 
Zhu, Jiang, Fuhong He, Songnian Hu, and Jun Yu. 2008. "On the Nature of Human Housekeeping Genes.” Trends in Genetics 24 (10): 481-84.

764

\section{$765 \quad$ Figure legends}

Figure 1. Number of expressed genes per cell type and the number of expressed cell types

767 per gene.

768 The number of expressed genes for each brain cell type (left) and the number of cell types one

769 gene was detected as expressed (right). The dotted vertical line on the left panel indicates the 2,368

770 commonly expressed genes.

\section{Figure 2. Gene and edge commonality distributions.}

773 The commonality of an edge indicates the number of cell types one edge was detected (top). The

774 commonality of a gene refers to the number of cell types one gene was detected as co-expressed

775 with at least one another gene (bottom). The y-axis shows the frequency of genes or edges in the

776 corresponding commonality score group, the numbers on top of each bar shows the counted

777 number of genes or edges. 
Figure 3. The observed edge commonality distribution compared with the null from network randomization.

The yellow dots show the observed edge commonality distribution and the grey dots the null distribution from network randomization. Network randomization was performed 100 times for each cell type individually with network size (number of nodes and edges) and gene degree

784 (number of co-expressed gene partners per gene) fixed. cutoff value defining a core.

A. Edge commonality was plotted against edge clustering coefficient. Edge commonality measures the number of cell types one edge was detected. Edge clustering coefficient shows one edge's neighborhood edge density.

B. The clustering coefficient values of the subgraphs (y-axis) change with increasing edge commonality cutoff values (x-axis). Progressive increasing edge commonality cutoff values were applied to the pan-network and edges whose commonality were equal or larger was calculated. 


\section{Figure 5. Decomposing the core subcellular network into modules.}

800

801

802

803

804

805

806

807

808

809

810

A. The heatmap of gene co-expression relationships and decomposed modules in the core cellular network. Modules which have at least 5 gene members are highlighted in different colors and numerically indexed. Gene symbols are shown on the right side of the matrix with colors matched to the corresponding module.

B. Network visualization of the core modules.

C. Enriched GO terms for each core module. We used the R package 'clusterProfiler' to perform gene set enrichment analysis of Gene Ontology with a Bonferroni correction and an adjusted $\mathrm{P}$ value cutoff of 0.05 . In each module, the top terms are shown (up to 6). A full list of enriched GO terms for each module is provided in Table S3.

\section{Figure 6. Distinct evolutionary signatures of core modules.}

A. We assigned fly genes into different evolutionary age groups in a phylostratigraphy framework. The number of genes in each evolutionary age group were compared between commonly expressed genes and genes constituting the core using a one-sided fisher exact test. Multiple-testing was adjusted using the p.adjust function in $\mathrm{R}$ with the Benjamini \& Hochberg method. $* * *$, p.adjust $<0.001$.

B. The age distribution of gene members of each core module. The size of each circle represents the proportion of genes in that evolutionary age group in the corresponding module. 

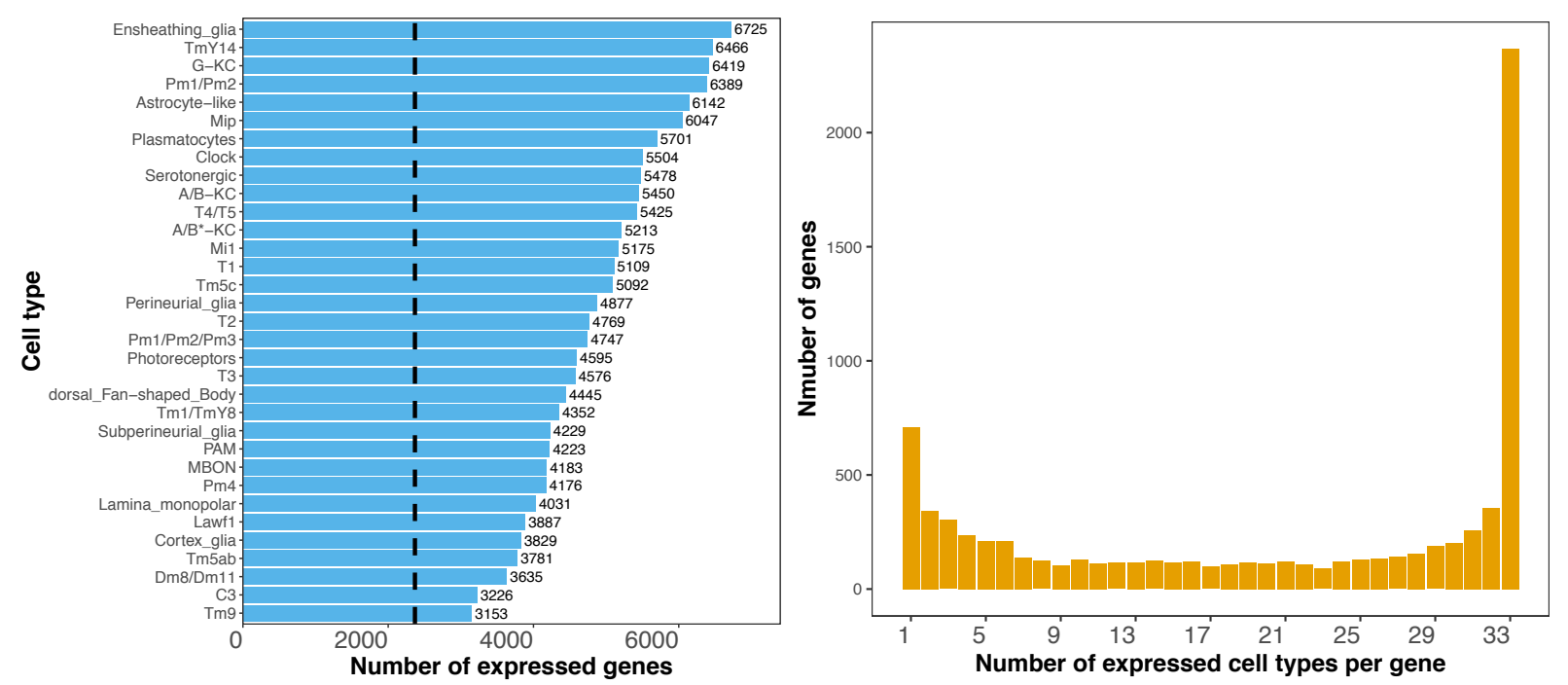

Figure 1. Number of expressed genes per cell type and the number of expressed cell types per gene.

The number of expressed genes for each brain cell type (left) and the number of cell types one gene was detected as expressed (right). The dotted vertical line on the left panel indicates the 2,368 commonly expressed genes. 

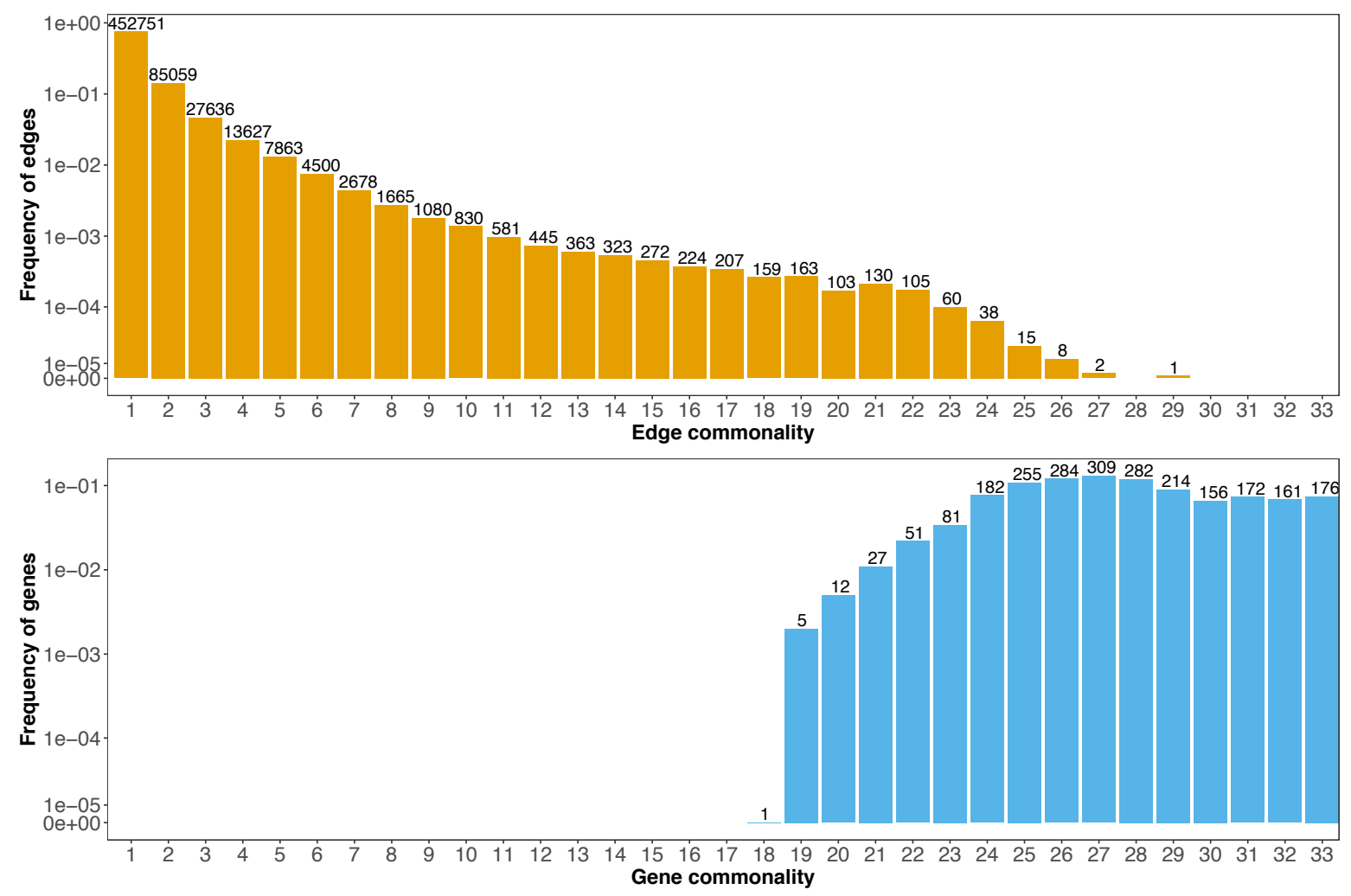

Figure 2. Gene and edge commonality distributions.

The commonality of an edge indicates the number of cell types one edge was detected (top). The commonality of a gene refers to the number of cell types one gene was detected as co-expressed with at least one another gene (bottom). The y-axis shows the frequency of genes or edges in the corresponding commonality score group, the numbers on top of each bar shows the counted number of genes or edges. 


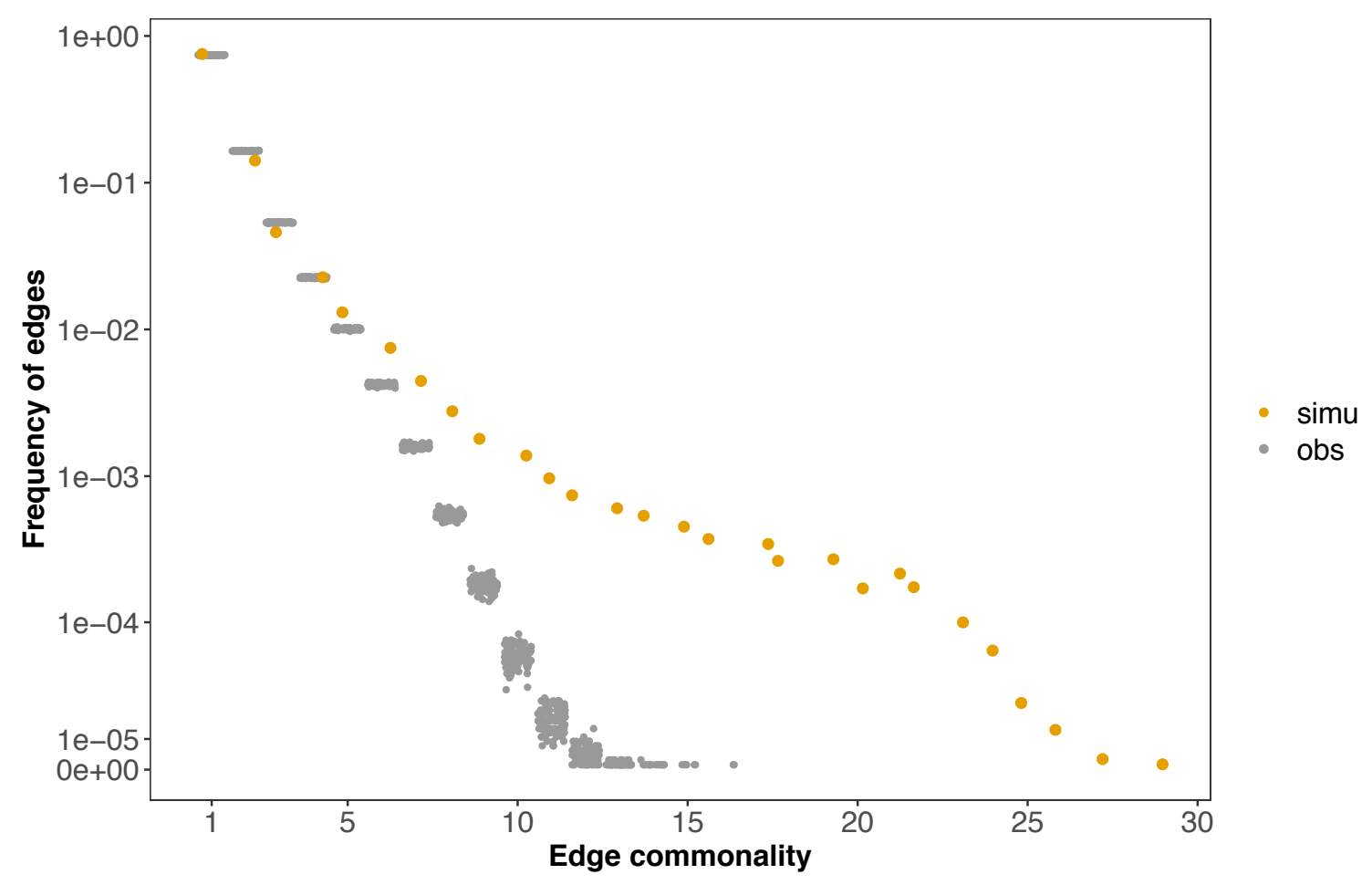

Figure 3. The observed edge commonality distribution compared with the null from network randomization.

The yellow dots show the observed edge commonality distribution and the grey dots the null distribution from network randomization. Network randomization was performed 100 times for each cell type individually with network size (number of nodes and edges) and gene degree (number of co-expressed gene partners per gene) fixed. 
A

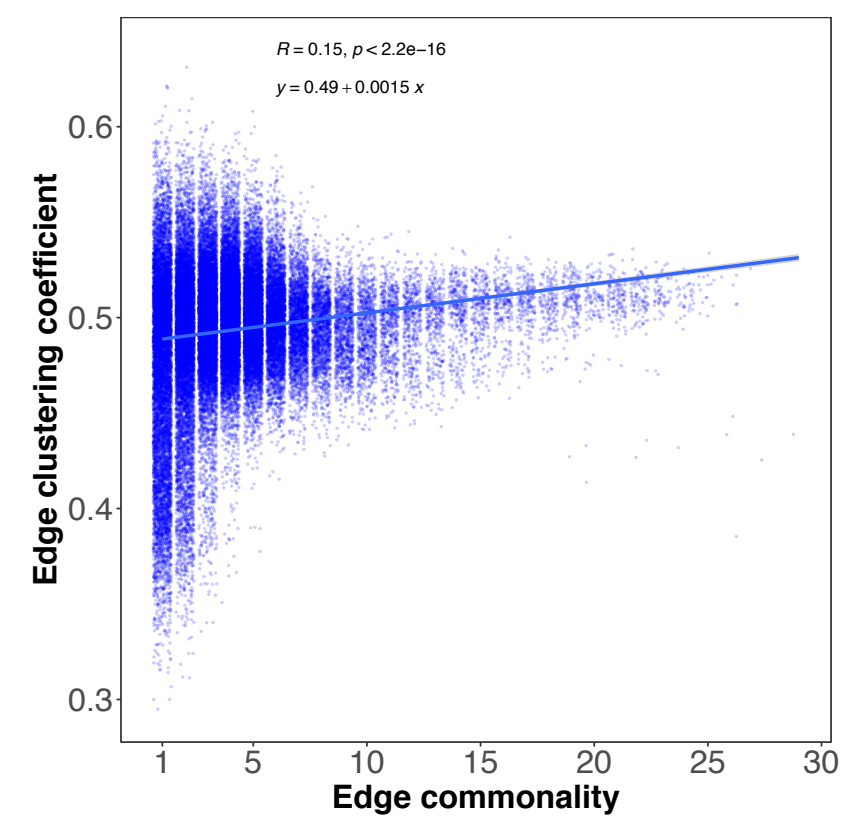

$\mathrm{B}$

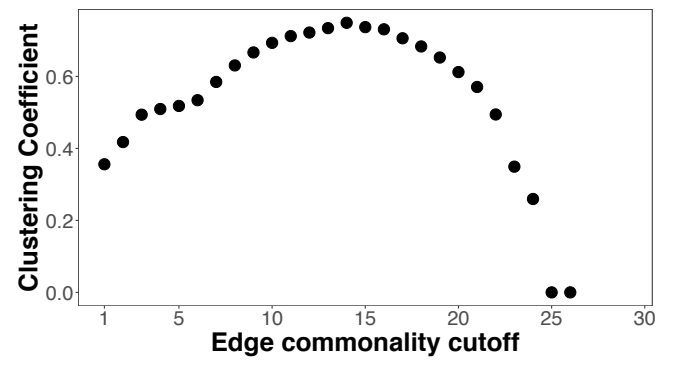

C status $\square$ core $\square$ non.core

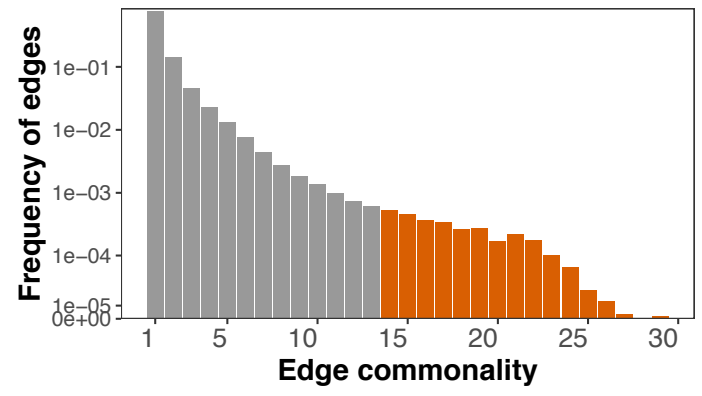

Figure 4. Topological properties of shared edges and the determination of edge commonality cutoff value defining a core.

A. Edge commonality was plotted against edge clustering coefficient. Edge commonality measures the number of cell types one edge was detected. Edge clustering coefficient shows one edge's neighborhood edge density.

B. The clustering coefficient values of the subgraphs (y-axis) change with increasing edge commonality cutoff values (x-axis). Progressive increasing edge commonality cutoff values were applied to the pan-network and edges whose commonality were equal or larger than the cutoff value were retained, the clustering coefficient of each resulting subgraph was calculated.

C. The edges composing a core cellular network at edge commonality cutoff 14 were highlighted in red. 
bioRxiv preprint doi: https://doi.org/10.1101/2021.09.19.460857; this version posted September 19, 2021. The copyright holder for this preprint (which was not certified by peer review) is the author/funder, who has granted bioRxiv a license to display the preprint in perpetuity. It is made available under aCC-BY-NC-ND 4.0 International license.

A
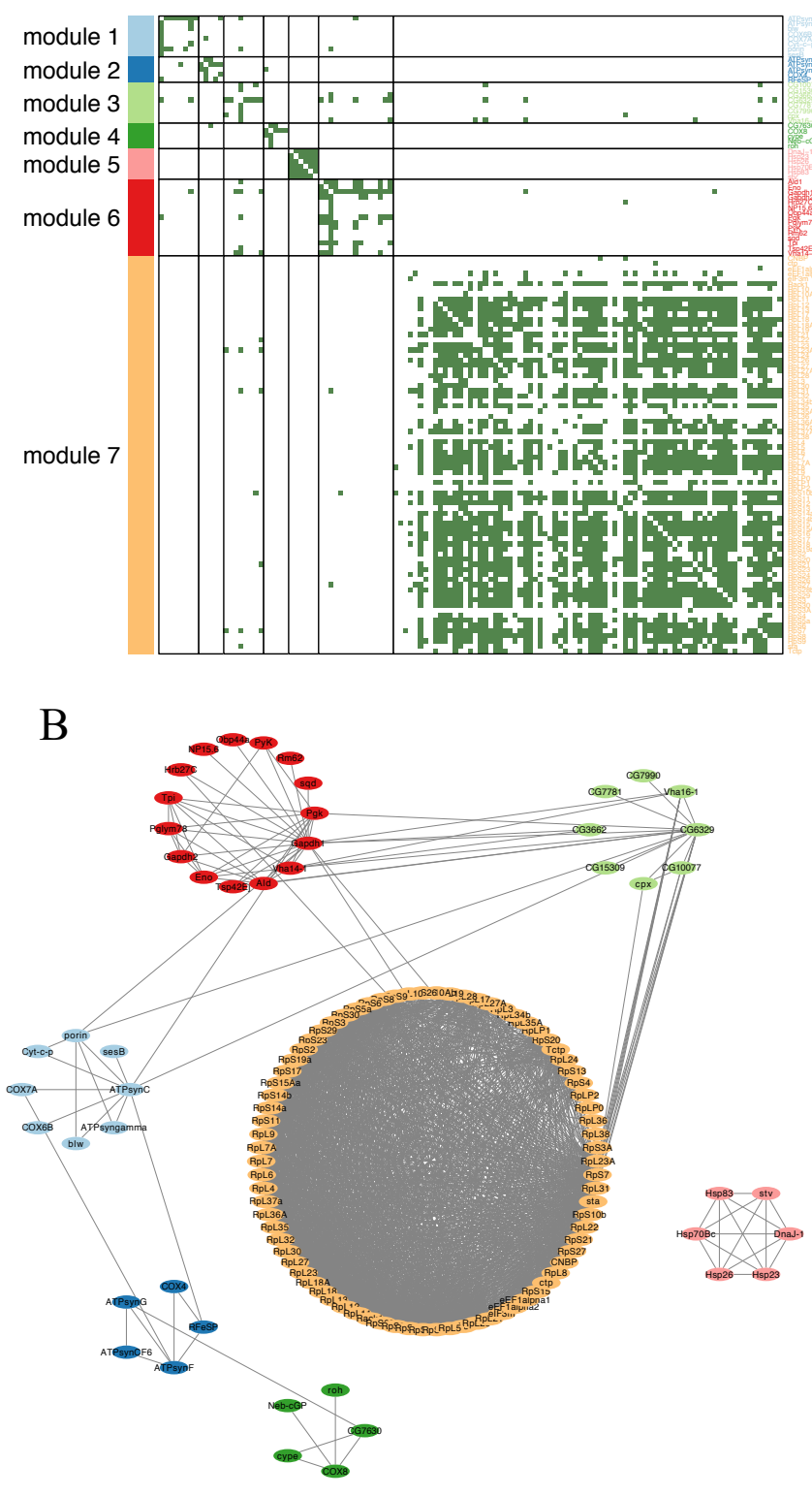

$\mathrm{C}$

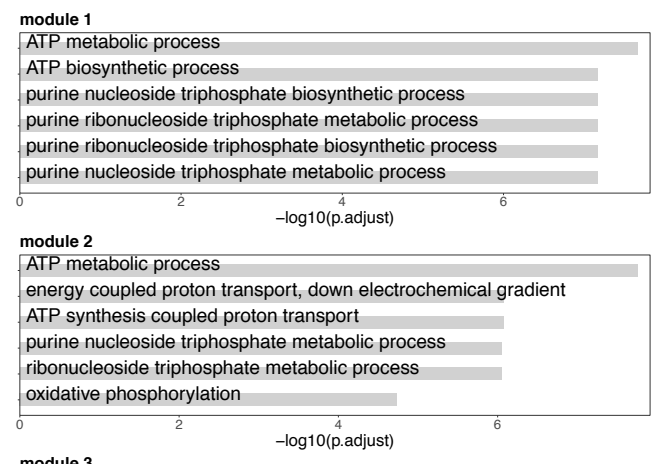

module 3

positive regulation of voltage-gated potassium channel activity regulation of synaptic transmission, cholinergic

positive regulation of potassium ion transmembrane transporter activity positive regulation of potassium ion transport

regulation of potassium ion transmembrane transporter activity

positive regulation of potassium ion transmembrane transport

module 4

$-\log 10$ (p.adjust)

rhodopsin biosynthetic process

aerobic electron transport chain

rhodopsin metabolic process

ATP metabolic process

$\begin{array}{rrr}0.0 & \\ 0.5 & -\log 10 \text { (p.adjust) } & 1.5\end{array}$

module 5

response to hea

protein folding

protein stabilization

positive regulation of cell differentiation

polytene chromosome puffing

regulation of crystal cell differentiation

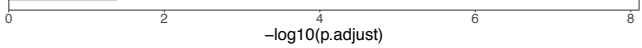

module 6

glycolytic process

ATP generation from ADP

purine nucleoside diphosphate metabolic process

purine ribonucleoside diphosphate metabolic process

ADP metabolic process

nucleotide phosphorylation

Jiation

-log10(p.adjust)

cytoplasmic translation

ribosome biogenesis

ribonucleoprotein complex subunit organization

rRNA processing

-log10(p.adjust)

Figure 5. Decomposing the core subcellular network into modules.

A. The heatmap of gene co-expression relationships and decomposed modules in the core cellular network. Modules which have at least 5 gene members are highlighted in different colors and numerically indexed. Gene symbols are shown on the right side of the matrix with colors matched to the corresponding module. 
B. Network visualization of the core modules.

C. Enriched GO terms for each core module. We used the R package 'clusterProfiler' to perform gene set enrichment analysis of Gene Ontology with a Bonferroni correction and an adjusted P value cutoff of 0.05 . In each module, the top terms are shown (up to 6). A full list of enriched GO terms for each module is provided in Table $\mathbf{S 3 .}$ 
A commonly.expressed.genes core genes

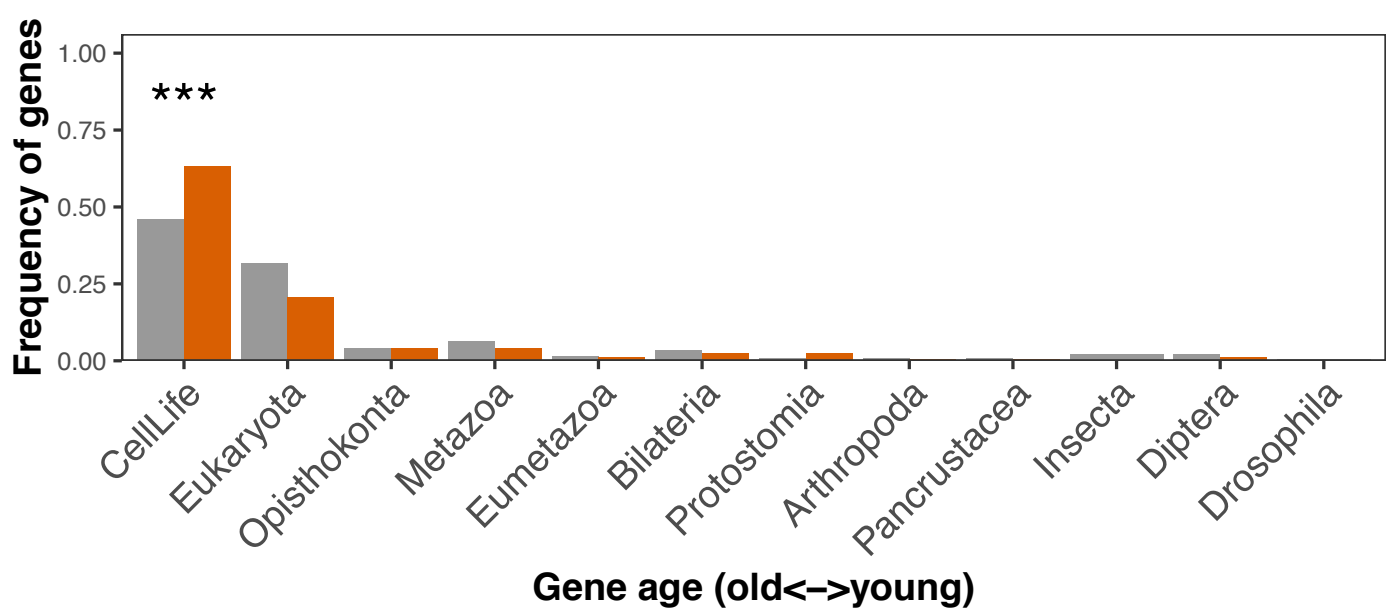

$\mathrm{B}$

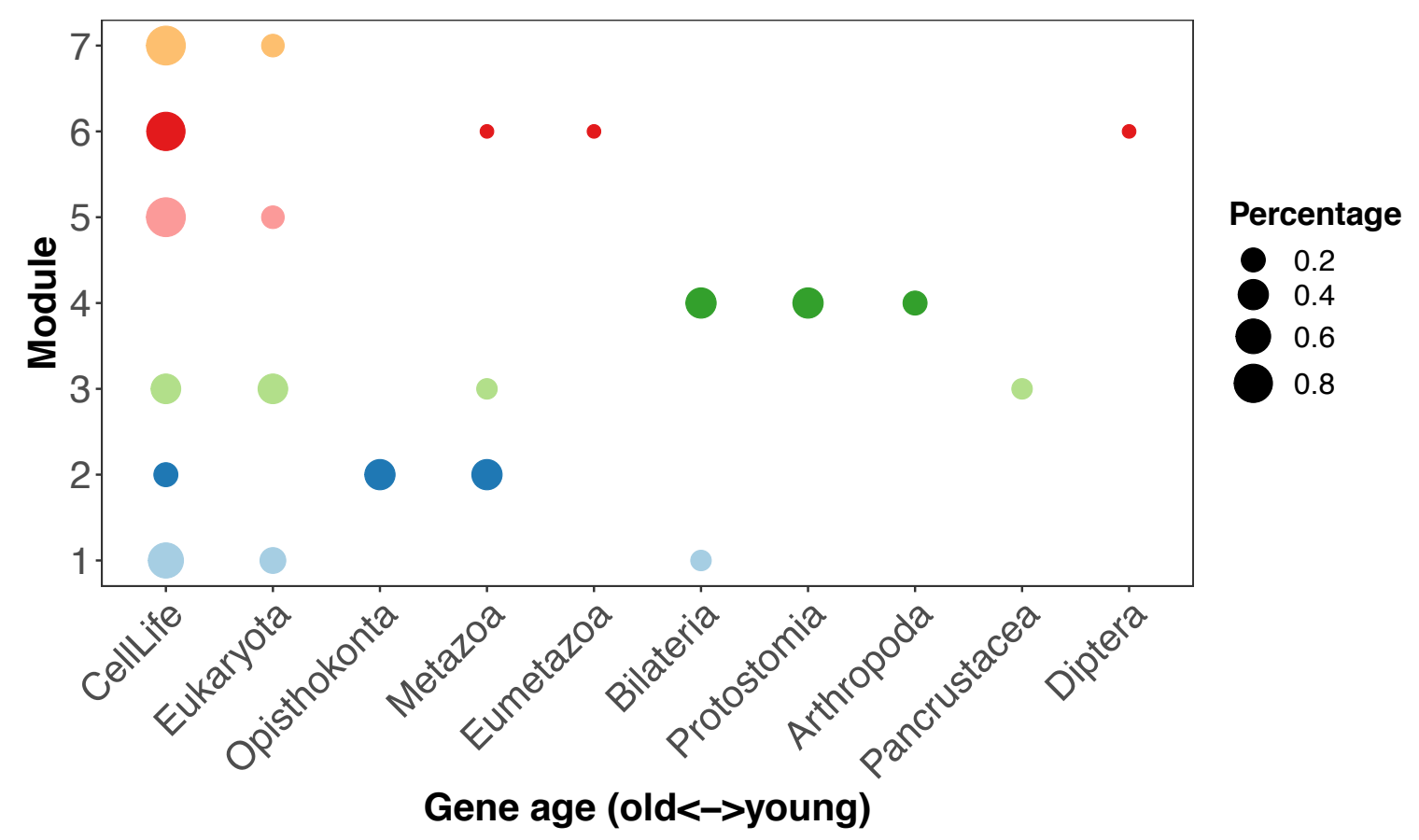

Figure 6. Distinct evolutionary signatures of core modules.

A. We assigned fly genes into different evolutionary age groups in a phylostratigraphy framework. The number of genes in each evolutionary age group were compared between commonly expressed genes and genes constituting the core using a one-sided fisher exact test. Multiple-testing was 
adjusted using the p.adjust function in $\mathrm{R}$ with the Benjamini \& Hochberg method. ***, p.adjust $<$ 0.001 .

B. The age distribution of gene members of each core module. The size of each circle represents the proportion of genes in that evolutionary age group in the corresponding module. 